\title{
DISTINCIÓN SOCIAL EN EL SENO DE LA BAJA NOBLEZA ARAGONESA: EL PALACIO DE LOS ZAPATA DE CALATAYUD EN 1484
}

\author{
SOCIAL DISTINCTION \\ IN THE LOW ARAGONESE NOBILITY: THE ZAPATA'S PALACE OF CALATAYUD (1484)

\section{GUILLERMO TOMÁS FACI \\ Universidad de Zaragoza}

\begin{abstract}
Resumen: En este artículo se analiza el inventario de los bienes existentes en la casa palaciega de los Zapata, linaje de escuderos asentado en Calatayud (Aragón, Península Ibérica), texto que se transcribe al final como apéndice. Este tipo de documentos permite conocer en profundidad el mundo material de las familias bajomedievales. La configuración del rico patrimonio mueble de los Zapata no dependía únicamente del desahogado nivel económico que disfrutaban, sino que respondía a una estrategia para reforzar los valores que les distinguían como miembros de la baja nobleza aragonesa y que, por lo tanto, justificaban su posición social dominante.
\end{abstract}

Palabras clave: Baja nobleza; Cultura material; Distinción; Palacio; Investario; Aragón; Siglo XV.

\begin{abstract}
This paper analyzes the goods inventory of Zapata's palace, a lineage of squirers installed in Calatayud (Aragon, Iberic Peninsula). The text is transcribed at the its end in Appendix. The inventories allow us to know deeply the material world the late medieval families. The configuration of the Zapata's rich movable patrimony did not only depend on the relieved economic level that they enjoyed but it responded to a strategy to reinforce the values that distinguished them like members of the low Aragonese nobility and that, therefore, justified their dominant social position.
\end{abstract}

Keywords: Low nobility; Material culture; Distinction; Palace; Inventory; Aragon; Fifteenth century.

\section{SUMARIO}

1. Introducción.- 2. La familia Zapata de Calatayud. 3. El palacio de los Zapata. 4. Los bienes propios de una familia de escuderos.- 5 . El nivel económico y de consumo.- 6 . Conclusión.

\section{INTRODUCCIÓN ${ }^{1}$.}

Más allá de la importancia que puedan tener las instituciones, las relaciones sociales o las mentalidades, resulta evidente que la cultura material

${ }^{1}$ Este trabajo hạ sido realizado gracias al disfrute de una Beca de Colaboración del Ministerio de Educación y. Ciencia durante el curso académico 2006-2007, en la Universidad de Zaragoza. El proyecto de investigación dentro del cual se enmarca este trabajo se centra en el estudio de una familia de la baja nobleza aragonesa, los Zapata, a través de la documentación conservada en el Archivo Histórico Provincial de Zaragoza (en lo sucesivo, AHPZ), entre la que se encuentra el texto sobre el que versa este artículo: AHPZ, Casa de Argillo, leg. 49, n⿳0 49. 
de una sociedad es fundamental para poder comprenderla. Sin embargo, la abundancia de fuentes documentales sobre la Edad Media ha reducido habitualmente el interés por los aspectos materiales de este periodo. ¿Qué medios tenemos para acceder al conocimiento de este elemento de las sociedades medievales? La arqueología es, en principio, el método esencial para adquirir esta información, aunque también se pueden citar otros procedimientos metodológicos, como la iconografía o, de manera mucho más general, el análisis de los objetos conservados en las colecciones públicas o privadas - generalmente estudiados por los historiadores del arte, bajo el epígrafe de "artes decorativas" u otros semejantes-. Las fuentes escritas medievales, especialmente en aquellos países que desarrollaron tempranamente la notaría, como Italia, el sur de Francia o Aragón, proporcionan otra importante vía de conocimiento, sobre todo a través de los inventarios post mortem, como el que presento aquí, que nos ofrecen una imagen bastante detallada de los bienes de una casa en el momento de la defunción de su propietario.

Las ventajas e inconvenientes de estos reflejos escriturarios del universo material que rodeaba a las gentes medievales son bastante evidentes y no es preciso insistir mucho en ello: por una parte, las descripciones son muy sumarias y apenas indican nada sobre las características o el valor de las piezas reseñadas; un adjetivo, algún diminutivo, en ocasiones apunta al deterioro o, por el contrario, al aprecio de estos objetos, pero no es lo usual. Por otra, los inventarios muestran la totalidad de las cosas que constituían el entorno de determinadas personas, que habían sido heredadas y elegidas por ellas, de modo que suministran indicaciones muy precisas sobre la sensibilidad individual de los propietarios, pero también sobre su inserción en la sociedad y, más allá de ésta, sobre la forma en que pretendían que esta posición fuese visible, la representación material de una identidad social. Algunas de estas cuestiones, a nuestro juicio, importantes puesto que inciden en problemas microsociales que son difíciles de abordar desde otros registros documentales, serán planteadas en las páginas siguientes a partir de un inventario concreto, el realizado en 1484 en la vivienda de Sancho Zapata, un escudero que residía en la ciudad aragonesa de Calatayud.

Los inventarios de bienes se podían realizar en diversas situaciones: dotes matrimoniales, embargos judiciales, repartos en herencia, etc. Sin embargo los más abundantes son los desarrollados después de la muerte de su propietario, generalmente en el comienzo de un periodo de tutoría o viudedad: su redacción era una garantía de integridad del legado transmitido a los legítimos herederos. En su realización participaba el notario, que certificaba la veracidad de los datos aportados, y los representantes de la familia. Eran más frecuentes cuanto mayor era la riqueza del patrimonio legado, pese a lo cual se hacían a menudo aunque no se tratasen de familias particularmente desahogadas: por ello, como se ha dicho, su estudio detallado permite observar las diferencias en la fortuna de las familias medievales.

Para el Aragón medieval se ha prestado escasa atención a este tipo de inventarios, y cuando se ha hecho ha tenido objetivos muy concretos, como 
el estudio del libro y los lectores a través del catálogo de bibliotecas ${ }^{2}$, o el estudio filológico ${ }^{3}$. Frente a esto, el número de inventarios editados es bastante notable y, entre ellos, destacan los setenta y cinco zaragozanos publicados por Manuel Serrano Sanz entre 1915 y 1922, o el formidable de la familia Sánchez Muñoz de Teruel, datado justamente en $1484^{4}$. Más recientemente, un artículo de $\mathrm{M}^{\mathrm{a}}$ Luz Rodrigo Estevan sobre la vivienda urbana en Daroca durante el siglo XV ha hecho uso de este tipo de documentos, aunque sin incluir su transcripción ${ }^{5}$.

Para trabajar sobre este tipo de documentos resulta fundamental tener buenos instrumentos de acercamiento al léxico romance bajomedieval, para poder relacionar los términos que utiliza la fuente y la realidad que representan. Para ello he recurrido tanto a estudios sobre el léxico medieval, como los de Sesma y Líbano, Pottier o Alonso ${ }^{6}$, como léxicos del aragonés actual ${ }^{7}$.

En este trabajo analizaré un inventario realizado en 1484 en Calatayud, destinado a catalogar los bienes muebles de una conocida familia de la nobleza local, con la finalidad de mostrar algunos aspectos de la vida cotidiana, el nivel de consumo y los hábitos propios de su rango social. Para ello empezaré por describir sucintamente la trayectoria y situación del linaje al que se refiere el texto, los Zapata; a continuación analizaré el espacio físico de una residencia nobiliaria que tiene características palaciegas: su situación dentro del casco urbano de la ciudad, su estructura y la función de sus diferentes ámbitos; por último me centraré en el ajuar de la vivienda, un indicador bastante objetivo para abordar los hábitos, la economía y el nivel de vida. El documento se añade como apéndice documental, puesto que sin duda un texto como este puede ser objeto de relecturas históricas o filológicas.

${ }^{2}$ Ángel SAN VICENTE PINO, Apuntes sobre libreros, impresores y libros localizados en Zaragoza entre 1549 y 1599, Zaragoza, 2003; José Manuel PEDRAZA GRACIA, Lector, lecturas, bibliotecas.... el inventario como fuente para su investigación histórica, "Anales de Documentación", 2 (1999): pp. 137-158.

${ }^{3}$ Bernard POTTIER, Étude lexicologique sur des inventaires aragonais, "Vox Romanica", 10 (1948-49): pp. 87-219.

${ }^{4}$ Manuel SERRANO SANZ, Inventarios aragoneses de los siglos XIV y XV "Boletín de la Real Academia Española", 2 (1915):pp. 85-97, 219-224, 341-352, 548-559, 3 (1916): pp. 89-92, 224225, 359-365, 4 (1917): 206-223, 342-355, 517-531, 6 (1919): 735-744, y 9 (1922): 118-134, 262-270; Curt J. WITTLIN, Un inventario turolense de 1484: Los Sánchez Muñoz, herederos del papa Clemente VIII, "Archivo de Filología Aragonesa", 18-19 (1976): pp. 187-215.

${ }^{5}$ María Luz RODRIGO ESTEVAN, La vivienda urbana bajomedieval: arquitecturas, conflictos vecinales y mercado inmobiliario (Daroca, siglo XV), "Stvdium. Revista de humanidades", 11 (2005): pp. 39-74.

${ }^{6}$ José Ángel SESMa MuÑoz y Ángeles LíBAno ZumalaCÁRREgui, Léxico del Comercio Medieval en Aragón (siglo XV), Zaragoza, 1982; POTTIER, Etude lexicologique; Martín ALONSO, Diccionario medieval español. De las Glosas Emilianenses y Silenses (s. X) hasta el siglo XV, Salamanca, 1986.

${ }^{7}$ Francho NAGORE LAÍn, Endize de bocables de l'aragonés: seguntes os repertorios lesicos de lugars y redoladas de l'Álto Aragón, Huesca, 1999. 


\section{LA FAMILIA ZAPATA DE CALATAYUD}

Los Zapata no eran una familia cualquiera de Calatayud: los Zapata presumieron a lo largo de su historia de ser uno de los linajes de más largo abolengo de la nobleza aragonesa, como intentaron poner de manifiesto en la Edad Moderna mediante la realización de varios memoriales y árboles genealógicos encaminados a demostrar la dignidad de su estirpe - con todos los beneficios que eso acarreaba- y perpetuar su memoria ${ }^{8}$. Por ejemplo, afirmaban descender de un hermano del rey Sancho Abarca, que había cambiado el apelativo "abarca" por su sinónimo "zapata" por tal de evitar confusiones, y haber estado presentes en los episodios fundacionales del mítico reino de Sobrarbe ${ }^{9}$. De esta familia de escuderos se conocen ramas radicadas en Zaragoza, Castilla y Valencia, y en lo que nos atañe, aparecen en la ciudad de Calatayud al menos desde mitad del siglo XIII. En el territorio de la Comunidad de aldeas de esta ciudad tuvieron en señorío los lugares de Valtorres y La Vilueña, que a final del siglo XIV pasaron a depender de los Luna por herencia, y poseyeron un castillo en Miedes, localidad que, sin embargo, fue de realengo desde un momento muy temprano.

La historia de nuestra rama de la familia Zapata, derivada de esta última fracción de la parentela, se puede trazar a través de la abundante documentación conservada en los fondos de los condes de Argillo, ahora en el Archivo Histórico Provincial de Zaragoza, aunque, como ocurre con frecuencia entre las familias nobiliarias aragonesas, la homonimia dificulta considerablemente la reconstrucción genealógica; en este caso, el hecho de que casi todos sus máximos representantes se llamasen Sancho Zapata plantea no pocos problemas. Durante la segunda mitad del siglo XIV, los Zapata aparecen junto a los Sayas, los Forcén o los Muñoz de Pamplona dentro del grupo de los Sayas, acerrimos enemigos de los Liñán, los dos bandos nobiliarios contendientes en la ciudad de Calatayud ${ }^{10}$; a lo largo de todo el siglo XV, el linaje mantendrá relaciones privilegiadas con los antiguos integrantes de dicho bando. En 1412 Sancho Zapata, habitante en Miedes, fue convocado y asistió a las Cortes de Zaragoza que juraron a Fernando $\mathrm{I}^{11}$, lo que indica su adhesión a la nueva dinastía reinante; poco después, en 1414, recibió una donación de la viuda de Gilberto Zapata, último miembro de la familia al que pertenecieron Valtorres y La Vilueña, consistente en un nutrido

\footnotetext{
${ }^{8} \mathrm{AHPZ}$, Argillo, leg. 49, no 40 contiene una historia de la familia y varios árboles genealógicos, y AHPZ, Argillo, leg. 71, $\mathrm{n}^{\mathrm{o}} 38$ es un memorial titulado "Nota de la antigüedad y nobleza de los Çapata de Calatayud". Ambos fueron realizados en época moderna.

${ }^{9}$ Sobre los legendarios "Fueros de Sobrarbe" y la ideología aristocrática implícita, puede verse: José Angel SESMA MUÑOZ, La creación de la memoria histórica. Una selección interesada del pasado, y Carlos LALIENA CORBERA, La apropiación mítica del pasado: poder real, legitimación y memorias de clase en Navarra y Aragon en el siglo XIII, en "Memoria, mito y realidad en la historia medieval. XIII Semana de Estudios Medievales" (2000), pp. 13-32 y 61-84.

${ }^{10}$ Vicente DE LA FUENTE, Historia de la siempre augusta y fidelísima ciudad de Calatayud, tomo II, Calatayud, 1881, pp. 21-30.

${ }^{11}$ Archivo de la Diputación Provincial de Zaragoza, ms. 11, fols. 5v y 54v.
} 
lote de tierras en Sabiñán ${ }^{12}$; en 1420 casó a su hijo Ferrán con María Jiménez de Sayas, proveniente de una rama del linaje aliado con ellos asentada en Sabiñán, lo que sugiere que estaban reforzando su presencia patrimonial en esta localidad. Ferrán Zapata encabezó el linaje en el transcurso del segundo cuarto del siglo XV, aunque las fechas son difíciles de establecer; se trata, por otra parte, del único personaje que llevó un nombre diferente al de Sancho en todo este periodo, un detalle que apunta a un acuerdo para que utilizase un apelativo propio de la familia materna. Su hijo, Sancho, se casó con Catalina de Bailo en las segundas nupcias de ella ${ }^{13}$ : él debió morir pronto, porque testó en 1452 y desapareció de la documentación, mientras que su viuda vivió, al menos, hasta que redactó testamento, en 1475. En dicho testamento el principal heredero vuelve a ser un Sancho Zapata, en el que cabe centrarse algo más, pues fue tras su muerte cuando se redactó el inventario de los bienes de la casa.

Sancho Zapata debió de nacer hacia 1450. Probablemente fue el hijo primogénito, por llevar el nombre característico de los patriarcas del linaje, lo cual le garantizó que su madre, Catalina de Bailo, lo convirtiese en su testamento en el heredero universal de todos sus bienes:

Item, quitas, pagadas, conplidas et exseguidas todas las lexas, mandas et ordenaciones en el present mi ultimo testament contenidas et expresadas, el residuo de todos mis bienes, assin mobles como sedientes, iura nomina debita a acciones, lexo al dito Sancho Çapata, fillo mio. En el qual residuo, heredero mio universal lo lexo, fago et constituezco con cargo que cumpla las lexas del present mi testament, et que lohe todas las lexas, mandas et ordenaciones en aquel contenidas et expresadas ${ }^{14}$.

Dicho texto lo hacía cargo de la mayoría de los bienes de la familia, además de obligarle a ocuparse de uno de sus hermanos:

[...] quiero et mando que mi heredero infrascripto aya de dar al dito mossen Pedro habitación franqua durant su bida en las casas do yo de present habito [...] tanto tiempo como yde querra habitar et morar $^{15}$.

Tomó como mujer a la donzella Leonor, hija del escudero Gonzalo Muñoz de Pamplona, lo que vuelve a mostrar la preocupación por establecer matrimonios con personas de similar rango social y mantener las tradicionales amistades de bando en el ámbito bilbilitano. Los capítulos matrimoniales fueron acordados en enero de 1467 por Catalina de Bailo y Gonzalo Muñoz de Pamplona: él aportó un importante patrimonio en propiedades, y ella una

\footnotetext{
${ }^{12}$ AHPZ, Argillo, leg. 2, no 29.

${ }^{13}$ En 1438 Catalina de Bailo figura en un documento (AHPZ, Argillo, leg. 49, no 103) como mujer del caballero Luis Forcen. De esta relación nació Violante Forcen, que a su vez tuvo cuatro hijos que aparecen en el testamento de su abuela, en 1475 (vid. nota 14).

${ }^{14} \mathrm{AHPZ}$, Argillo, leg. 49, $\mathrm{n}^{\mathrm{0}} 93$.

${ }^{15}$ Idem.
} 
dote de 8.000 sueldos $^{16}$. Los hijos del matrimonio, Sancho, Gonzalo, Juan, Isabel y Catalina, debieron tardar en llegar, porque en el momento de confeccionar el inventario ninguno parece haber alcanzado la mayoría de edad de catorce años.

Sancho Zapata otorgó testamento el día 11 de abril de 1484, dos semanas antes de que se redactase el documento que nos interesa, por lo que entre esas dos fechas hubo de producirse su muerte ${ }^{17}$. En él declara herederos a sus cinco hijos, y señala los nombres de los tutores, los mismos que se citan al comienzo del inventario. El notario Juan Cortés realizó dicho inventario entre los días 24 y 26 de abril de 1484, por orden y en presencia de los tutores y albaceas testamentarios de los cinco hijos: Juan e Isabel, hermanos del difunto, y Gonzalo Muñoz, padre de Leonor, aunque este último no pudo acudir al estar enfermo, por lo que figuran dos miembros de su familia en su lugar. La finalidad de este documento era conocer con exactitud los bienes legados por los padres, para que fuesen correctamente transmitidos a sus legítimos herederos al cabo de su minoría de edad. Así se describe en el texto:

Por tanto, en absencia del dicho Goncalvo Munyoz, contutor con ellos, dixeron que por satisfacer al Fuero, presentes mi notario y testimonyos infrascriptos, procidian y procedieron a fazer y fazieron inventario de los bienes mobles del dicho Sancho Capata, quondam, que stavan en las casas de su habitación et se trobaban et trobaron dentro de aquellas al tiempo de su muerte.

El conocimiento de la economía de la familia Zapata exigiría un esfuerzo suplementario y la localización de nuevas fuentes, pero la documentación que he consultado en el Archivo Histórico Provincial de Zaragoza para el siglo XV proporciona algunos elementos de juicio. A lo largo del siglo XV no he localizado a ningún miembro de la familia Zapata de Calatayud al frente de un señorío jurisdiccional, aunque sí sabemos que obtuvieron algún beneficio propio de su categoría más allá de las ventajas fiscales: el noble Juan de Luna concedió en 1421 a Sancho Zapata - probablemente el mismo que había jurado fidelidad a Fernando I - una caballería de honor, con una renta de quinientos sueldos anuales a pagar por la Comunidad de aldeas de Calatayud, a cambio de servir:

[...] a nos e al senyor Rey toda vegada que requerido en seredes con un cavallo armado segunt Fuero, Uso e Costumbre del Regno de Aragón ${ }^{18}$.

Los Zapata debieron de basar su riqueza en la explotación de las tierras que poseían en varios lugares de la Comunidad de Calatayud como Miedes, Belmonte, Sabiñán o la propia ciudad, aunque las frecuentes transformaciones de este patrimonio por las dotes de los matrimonios impiden

\footnotetext{
${ }^{16}$ AHPZ, Argillo, leg. 49, no 108.

${ }^{17}$ AHPZ, Argillo, leg. 49, no 59.

${ }^{18} \mathrm{AHPZ}$, Argillo, leg. 49, no 120.
} 
precisar con seguridad las propiedades que tenía Sancho Zapata antes de 1484 . Según indican los documentos, la mayoría de las tierras estaban dedicadas a la viña y al cereal, y en menor medida, al olivar y el zumaque -planta de la que se obtenía un tinte azulado de mediana calidad muy utilizado en la industria textil aragonesa-. Este conjunto de propiedades no era, evidentemente, explotado directamente, sino que se recurría a contratos de explotación agraria por periodos breves de cinco años o "collidas", según se puede observar en dos ejemplos conservados de 1415 y $1416^{19}$. En las capitulaciones matrimoniales de 1467 también figura un molino en la localidad de Belmonte de Gracián, que debía proporcionar ingresos constantes a la familia ${ }^{20}$.

Aunque los testimonios aportados son más bien escasos, parecen indicar que no se trataba de una familia de la baja nobleza especialmente adinerada, cuyos ingresos se basaban en el arriendo de sus tierras, pero mantuvo estable su situación a lo largo del periodo estudiado, realizó enlaces matrimoniales con otras familias prestigiosas $-\mathrm{y}$ tal vez con mayores medios de fortuna- como los Muñoz de Pamplona, los Sayas o los Santángel ${ }^{21}$, y disfrutó de ciertas comodidades y lujos en su morada de Calatayud, como espero demostrar a través del inventario de sus bienes.

\section{El PALACIO DE LOS ZAPATA}

En la introducción que el notario escribió al inventario de los bienes de Sancho Zapata, se describe brevemente la situación de sus casas:

Las quales dichas casas son sitias en la dita ciudat de Calatayut, que afruentan con casas de Joan de Puydenina, con casas de Pero Tris, notario, et con casas de herederos de don Gostin de Savinyan, quondam.

El citado testamento de Catalina de Bailo aporta datos más concretos para situarla en el plano de la ciudad, en el mismo párrafo en que se obligaba a Sancho a mantener a su hermano en ella mientras éste quisiese:

[...] en las casas do yo de present habito, que son sitias en la Ruva de la dita ciudat, que afruenta con casas de Luys de Savinyan, con casas de Johan de Puydenina e con la Ruva $[\ldots]^{22}$.

De esto se deduce que las casas de la familia Zapata se situaban en una de las calles más céntricas e importantes de la ciudad aún hoy: la Rúa de Dato. Entre su vecindario debían de contarse otras familias importantes que escogieron este espacio emblemático para instalar sus casas, como muestra el

\footnotetext{
${ }^{19} \mathrm{AHPZ}$, Argillo, leg. 2, no 32; AHPZ, Argillo, leg. 70, $\mathrm{n}^{\mathrm{o}} 29$.

${ }^{20}$ Vid. nota 16.

${ }^{21}$ Con mujeres de la familia Sayas se casó Ferrán Zapata en 1420, y lo hará en 1494 Sancho Zapata; por su parte, su hermano Pedro se casó en 1491 con Isabel de Santángel.

${ }^{22}$ AHPZ, Argillo, leg. 49, no 93.
} 
que uno de sus vecinos fuese notario. Aunque nunca las llamen así, éstas debían de ser las "casas mayores" de los Zapata, en las que residieron varias generaciones de herederos de este linaje. Por ello, los objetos que se encontraban en ella en 1484 no deben interpretarse como las adquisiciones del matrimonio de Sancho y Leonor únicamente, sino como el resultado de la acumulación de bienes de varias generaciones de habitantes del edificio ${ }^{23}$.

En 1963 se derribó el número 5 de dicha calle de Calatayud, un edificio palaciego llamado popularmente "Casa de Argillo", de la que sólo se conservó - desmontado- el alero renacentista que coronaba su fachada ${ }^{24}$. El hecho de que el linaje de los Zapata desembocase en el de Argillo -como muestra que sus documentos privados se hayan incorporado al archivo de dicha familia-, y la coincidencia del emplazamiento, abren la puerta a identificar el edificio que se analiza aquí con el desaparecido palacio.

La organización física de la casa puede imaginarse a partir de la descripción que ofrece el inventario: este tipo de instrumentos notariales solía realizarse estancia por estancia, lo que nos permite conocer los ambientes de que disponía. También es muy útil recurrir a otros trabajos realizados sobre la vivienda urbana aragonesa durante el periodo que nos ocupa ${ }^{25}$. Actualmente se conservan algunos restos de arquitectura civil de este periodo en la ciudad, aunque su grado de enmascaramiento dificulte sacar conclusiones al respecto; tal vez el llamado Mesón de la Dolores sea el edificio mejor conservado ${ }^{26}$. Fuera de Calatayud, la casa de la calle las Armas n ${ }^{\circ} 32$ de Zaragoza es un buen ejemplo de los palacetes urbanos en los que residían los grupos acomodados aragoneses ${ }^{27}$.

El inventario comienza describiendo un palacio situado frente a la puerta de entrada en el edificio, la habitación en la que se encuentran más objetos, luego pasa al porche, y después relaciona el contenido de otras tres piezas: una cambra y dos cambretas; aquí acaba la descripción realizada el primer día, 24 de abril. Dos días después sigue el inventario por dos retretas de la sala, para pasar después a la sala, una cambra en la que se acumulan numerosísimos objetos, la cozina y el reposte, la bodega y acaba de nuevo en el porje. Analizar la organización espacial de la casa a partir de esta somera descripción es complicado, ya que no se señalan las habitaciones en las que no había objetos inventariables, como puede ser el retrete o la falsa, ni se mencionan elementos tan importantes como es la chimenea, por estar

\footnotetext{
${ }^{23}$ Con los datos de que disponenemos, se puede afirmar que al menos vivieron en ella tres generaciones: Catalina de Bailo, Leonor Muñoz de Pamplona e Isabel de Santángel, con sus respectivos maridos llamados Sancho Zapata.

${ }^{24}$ Carmen Gómez URDÁÑEZ, El alero del llamado Palacio de Argillo de Calatayud, en “Actas del I Encuentro de Estudios Bilbilitanos", tomo 1 (1982), pp. 179-191.

${ }^{25}$ Carmen GómEZ URDÁÑ̃Z, Arquitectura civil en Zaragoza en el siglo XVI, Zaragoza, 1987; RoDRIGO, La vivienda urbana medieval.

${ }^{26}$ Agustín SANMIGUEL MATEO: Un alfarje del siglo XV en Calatayud, en "Actas del III Encuentro de Estudios Bilbilitanos", tomo 1 (1982), pp. 303-309; Agustín SANMIGUEL MATEO y Ana Isabel PÉTRIZ ASO El Mesón de la Dolores, en "Actas del TV Encuentro de Estudios Bilbilitanos", tomo 1 (1997), pp. 385-391.

${ }^{27}$ GómEZ, Arquitectura civil en Zaragoza, pp. 164-166.
} 
incorporada en la obra -aunque su equipamiento puede ayudarnos a localizarla-, ni tampoco se especifica la distribución en alturas. Posiblemente el primer día analizó las estancias de la primera planta, incluyendo el palacio o sala baja, y al día siguiente se centró en las de la planta superior, con la sala propiamente dicha.

La vivienda dispondría probablemente de tres alturas y una bodega: la inferior, que se abría a la calle a través del porche, la principal, en las que se encontraban las habitaciones que más debían frecuentar los habitantes, y una falsa superior que, aunque no es mencionada en el inventario, la necesidad de aislamiento térmico la haría prácticamente imprescindible; la bodega excavada sería similar a la que se conserva bajo el Mesón de la Dolores, y su construcción hubo de acompañar la orientación de la economía familiar hacia la viticultura. La fachada a la Rúa sería sencilla: una galería superior como se estaba generalizando en la arquitectura civil, ventantas no muy amplias para no perder calor, una puerta; algún elemento, tal vez las armas de la familia, indicaría al viandante o el huésped quiénes vivían en esa casa. Al interior debía haber una luna o corral para iluminar las estancias que no se abrían a la calle. Probablemente el aspecto general de la casa sería un poco caótico, fruto de las sucesivas ampliaciones del edificio original, pues hasta el siglo XVI fueron escasas las reconstrucciones en el tejido urbano aragonés.

El porche debía de ser el espacio cubierto existente nada más atravesar la puerta de entrada en el edificio, a partir del cual se accedía al resto de las estancias. En él había sendas alfaceras (una especie de esteras) en la pared y en el suelo, una pequeña artesa para dar de comer a los animales domésticos y varios sacos destinados, quizás, al almacenaje de algún producto agrícola.

La palabra palacio tenía en la Baja Edad Media un sentido más amplio del que posee actualmente: a veces eran estancias auxiliares, pero frecuentemente designaba a la sala baja de un edificio, como en nuestro caso. De hecho parece ser la estancia más noble de la casa, mucho más que la sala propiamente dicha. La existencia de dos habitaciones principales ha de responder a funciones diferentes: la sala baja sería más utilizada con invitados y en verano, pero su situación en la planta baja la haría menos acogedora durante los largos meses de frío, mientras que la sala, junto a los dormitorios, estaría más destinada a la vida familiar y cotidiana. El mobiliario del palacio se podría resumir así: dos camas, una muy equipada que tenía un banco al costado, aislada por un paramento (dosel) de cinco cortinas, y otra de reposte, donde duermen mocas ${ }^{28}$; hay dos mesas de madera, cinco cadiras o sillas, y un escabel; la gran mayoría de los objetos se distribuyen dentro de cinco cofres y una caja de pino. Las catifas y la alhombraca debían de estar en el suelo y en un caso se especifica que estaba destinada a sentarse sobre ella; las paredes se adornaban con cortinas, un tapiz con las armas de la familia Zapata

${ }^{28}$ La referencia a las mọcas o sirvientas resulta de gran interés, porque muestra la existencia en algún momento de servicio doméstico residiendo en el edificio. 
y una spada vieja colgada; para iluminar había un candelero colgado de fusta en medio.

Como se ha dicho, la riqueza del palacio contrasta con la austeridad de la sala: en ella sólo aparece un banco y un parador, en el que debían de estar las toallas, sábanas y manteles que se mencionan. A esta sala se abrirían las dos retretas de la sala que menciona el inventario, que podrían ser sendos dormitorios: en una sólo hay una cama entaulada; en la otra hay una cama, un retablico de fusta pintado, una mesa y un banco, y como decoración otro paño con las armas de la familia. Por el mobiliario y ubicación, la segunda retreta debía ser el aposento privado de Sancho Zapata. Hay dos habitaciones más, denominadas cambretas que, por el equipamiento, hubieron de desempeñar la función de dormitorios: en una de ellas hay tres cajas que contenían algunos objetos de valor de oro, plata y marfil, y algo tan cotidiano como unas patoflas viejas.

La cozina era una habitación aparte: el hecho de que no fuese una de las habitaciones más importantes de la casa parece mostrar el alejamiento de los propietarios de las labores domésticas. El mobiliario no era muy abundante: un parador, una mesa y dos bancos; el instrumental de cocina es escaso en esta pieza, y posiblemente en el momento del inventario estaba en su mayor parte almacenado en la cambra que se describe antes de la cocina, donde aparece en gran abundancia. Después se indica la existencia de un reposte, que posiblemente sería una pequeña habitación adjunta a la cocina, en el que se almacenaban dos toneles de vino, un cofre para guardar pan, y cuatro tinajas, tal vez relacionadas con el almacenamiento de agua.

Como he indicado, entre la cozina y la sala se describe una cambra cuyo mobiliario es tan abundante y dispar que en el momento en que se realizó el inventario debía de estar destinada a almacén. En ella está el conjunto más importante de utensilios de cocina y equipamiento de la chimenea, seguramente relacionados con la cocina que se acaba de mencionar; también hay bastante utillaje militar, tanto armamento como la montura de un caballo; figuran también objetos muy diversos, como varios textiles, una cuna, dos mesas - sin más asiento que una silla vieja - y algunos candeleros.

Quedan por describir dos habitaciones relacionadas con las actividades que se desarrollaban en la casa. En primer lugar aparece una cambra en la que hay mobiliario para fabricar pan, algunos instrumentos de cocina y dos mesas de reparar: seguramente es lo que en otras ocasiones se denomina "masadería". Por último, en la bodega está todo lo relacionado con la fabricación y almacenamiento del vino.

Dentro de cada una de las estancias la mayoría de los objetos de reducidas dimensiones se distribuía en el interior de diferentes cofres y cajas, mientras que sólo se menciona un almario y un parador; la caja que contiene los objetos más valiosos se encuentra en una de las cambras. Para lograr la confortabilidad del edificio era preciso tener un buen número de paños, raceles, catifas y alfombras que aislaban el suelo y paredes del frío exterior. Para cerrar las puertas y ventanas, o para configurar ambientes singularizados dentro de las estancias, se utilizaban cortinas, paramentos y tancaportas, en 
algún caso decorados. La iluminación natural debía de ser escasa, puesto que los vanos eran un escape del calor; la luz artificial era proporcionada por candeleros, que podían ser móviles o instalados en el techo. El calor era proporcionado por una chimenea que, si bien el inventario no hace explícita, existió con seguridad y tenía un completo equipamiento: asaderas, trehudes, fogariles, porpalo, llares...

La estructura de la casa demuestra que pertenecía a un grupo social acomodado: hay una neta separación entre las habitaciones dedicadas a las actividades domésticas, seguramente frecuentadas por el personal que se encargaba de estas tareas, y las estancias más nobles destinadas a la vida familiar e incluso a la ostentación de las armas del linaje o de una vieja espada. Aunque de manera algo imperfecta, se observa también una distinción entre las estancias destinadas a dormir y a la actividad personal de cada uno de los habitantes de la casa - las cambretas o retretas, cuyo principal mobiliario es siempre una cama-, las salas comunes donde se desarrollaba la vida familiar, e incluso una sala destinada a la actividad social, la más próxima a la entrada desde la Rúa, posiblemente visible desde ella.

\section{LOS BIENES PROPIOS DE UNA FAMILIA DE ESCUDEROS}

El análisis de los objetos de la familia Zapata se corresponde perfectamente con el estatus de escudero que habían disfrutado numerosos de sus miembros. Escudero era una de las categorías que formaban parte del amplio grupo de la baja nobleza aragonesa; la legislación foral definía algunos de los rasgos de este estamento, como podía ser su dedicación, si era necesario, a actividades guerreras, o las numerosas exenciones fiscales de las que disfrutaban ${ }^{29}$. Los Fueros de Aragón, en su libro séptimo, dicen que "el Creador del mundo [...] ordenó que todos y cada uno de los hombres tuvieran precisos y distintos órdenes", de manera que "los caballeros sean siempre defensores", y "los demás hombres cumplan cada uno su oficio", lo que refleja el rechazo del trabajo manual de este colectivo ${ }^{30}$, lo que no quiere decir que no estuviesen vinculados a tareas administrativas dentro del estado bajomedieval, o al notariado, entre otras que consideraban apropiadas.

El inventario muestra que el palacio de los Zapata cumplía funciones esencialmente residenciales, y carecía del equipamiento productivo ajeno a las actividades propiamente domésticas, como las relacionadas con la producción del pan, desarrolladas en la habitación que cumplía las funciones de masadería, o el textil, visible por la presencia de dos restillos (peines de lino) y una devanadera. No aparece ningún tipo de estancia relacionada con actividades artesanales ni, aparentemente, con la venta al público de algún producto. Respecto al equipamiento agrícola, que en principio debería figurar entre los

\footnotetext{
${ }^{29}$ Pascual Savall y Santiago PenÉn, Fueros, Observancias y Actos de Corte del Reino de Aragón, tomo 3, Zaragoza, 1999, pp. 135-148.

${ }^{30}$ Ídem, p. 139.
} 
bienes de unos importantes terratenientes, no aparece ni siquiera lo mínimo, una circunstancia que apunta a que todos estos instrumentos se encontraban en las casas o torres que centralizaban las explotaciones agrícolas, o habían sido cedidos a los cultivadores mediante acuerdos de préstamo de herramientas, vinculados a la cesión de las tierras a cambio de rentas. De este modo, no aparece más que una legona (media luna), en la cambra en la que se almacenaban objetos muy diversos, y algún objeto relacionado con el transporte y almacenamiento, aunque esto no tiene por qué suponer actividad agrícola alguna: unas arguinas (una especie de alforja que se colgaba del hombro), en la misma cambra, y un par de sacos en el porche.

La única habitación estrechamente relacionada con una actividad productiva es la bodega, en la que se sitúan todos los objetos relacionados con el vino. Allí hay onze cuevanos de vendemar, que no debieron de ser utilizados por los Zapata para recoger los frutos de sus numerosas vides dispersas en campos de Calatayud y otros pueblos de la comarca, sino para organizar el trabajo de un grupo de personas a sueldo que vendimiarían en su lugar. En la bodega también había un tino grande et onze cubas, las nueve grandes, de las quales havia quatro grandes plenas de vino tinto, es decir, que había una importante cantidad de vino almacenado que rebasaba las posibilidades de consumo familiar: probablemente se utilizaría esta bodega para guardar y comercializar el producto, del mismo modo que documenta $\mathrm{M}^{\mathrm{a}}$ Luz Rodrigo en una familia de escuderos de Daroca ${ }^{31}$. A diferencia de la gestión de los campos de cereal, cuyo fruto no era en absoluto conservado en graneros o zonas específicas de la casa, parece que la explotación de algunas viñas de los Zapata fue organizada directamente por la familia, y el producto resultante se almacenaba en la bodega y se vendía en el mercado urbano de Calatayud, constituyendo otra fuente de ingresos.

La presencia de armamento en una vivienda no es sinónimo automático de la pertenencia a un grupo social acomodado y nobiliario, aunque la calidad y cantidad que detectamos en este caso sí resulta algo característico de una familia de escuderos. Este es el equipamiento militar que figura en el inventario: dos fajas pora ombres d'armas, tres ballestas de fusta, una ballesta de azero, dos pares de cubiertas de cavallo, unas curacas blancas, unas curacas guarnecidas de negro, unos gozetes, una cervellera, un guante de malla, una espada guarnecida de negro, un fierro de lança, una aljava con nueve passadores, diez pavesses pintados y un dargon. La cantidad y diversidad de elementos obligan a pensar en el equipamiento de un pequeño grupo militar dependiente y organizado por los Zapata: dos personas a caballo -seguramente el patriarca y su heredero- y un grupo de hasta cuatro ballesteros a pie, que se reclutaría entre la clientela del linaje. En el momento de realizar el inventario este grupo militar debía estar inactivo, lo que explica que todo el material bélico se acumulase en un almacén junto a objetos diversos, pero la concesión de la caballería de honor en 1421, el fondo de

\footnotetext{
${ }^{31}$ RoDRIGO, La vivienda urbana bajomedieval, pp. 60-61.
} 
violencias urbanas que se remontan al siglo XIV y la consciencia de unas funciones sociales singulares, demuestran que como escuderos estaban preocupados por el ejercicio de la violencia. La dedicación guerrera era algo más que una obligación para los Zapata: era un modelo de vida, un conjunto de valores que los singularizaban, y que debían publicitar adecuadamente para mantener el prestigio social que poseían. En 1434 el caballero leonés Suero de Quiñones desafió a todo aquel que quisiese atravesar el río Órbigo, camino a Santiago de Compostela; el desafío caballeresco fue cuidadosamente preparado, como era habitual en estos casos. Cinco bilbilitanos aceptaron tan peculiar reto militar: Rodrigo de Sayas, Francisco Muñoz, Fernando de Liñán, Antón de Funes y Sancho Zapata ${ }^{32}$. Nuestro escudero corrió diecinueve lanzas y rompió tres, y lo más importante, logró que su gesta pasase a la historia de la caballería, y que su nombre ganase en respeto y prestigio. Ésta y otras historias, con todo el código cultural que contienen, serían transmitidas a hijos, nietos y visitantes, y la spada vieja colgada que presidía el palacio cumpliría una función didáctica fundamental en ese proceso. Semejante objeto debía estar cargado de un valor simbólico evidente y concreto para los Zapata, y en cierto modo era una justificación de un estatus diferente y privilegiado.

En la Edad Media la casa era "símbolo de unidad de los clanes familiares" 33 , y también el símbolo del linaje que la ocupaba: por ello se entiende que en el inventario hubiera numerosos objetos que mostraban las armas de la familia. El escudo de armas fue desde la Edad Media la plasmación gráfica más evidente del linaje: el de los Zapata consistía en tres zapatos en oro sobre fondo rojo ${ }^{34}$; en el inventario aparecen siete objetos diferentes que lucían las armas de los Capatas: suelen ser elementos muy visibles, como sendos trapos de paret, dos tancaportas o una sobremesa, o lujosos utensilios que hubieron de ser realizados por encargo, como un salero d'argent o un sillo (sello) de oro. También estaban presentes en la decoración las armas del rey de Aragón, expresión de sometimiento a su soberanía, y también de proximidad a su poder. La ostentación de los signos externos de la categoría social es especialmente palpable en el palacio que hemos descrito, seguramente la habitación en la que se recibía a los invitados ajenos a la familia: sobre una pared estaba la vieja espada; también sobre la pared se mostraba uno de los paños con las armas de la familia. Por lo tanto en la casa había objetos con gran valor simbólico, estratégicamente situados para que fuesen percibidos por el invitado, que mostraban que sus habitantes pertenecían a un importante linaje de escuderos.

El valor testimonial de la escritura también fue apreciado por esta familia, que guardaba un talegon de cuero pleno de cartas y scripturas, que

\footnotetext{
${ }^{32}$ Fernando ARROLLO ILERA, Libro del Passo Honroso, defendido por el excelente caballero Suero de Quiñones, Valencia, 1970, pp. 13-16; Martín DE RIQUER, Caballeros andantes españoles, Barcelona, 1967, pp. 54-99.

${ }^{33}$ Jacques HEERS, El clan familiar en la Edad Media, Barcelona, 1978, pp. 124-126.

${ }^{34}$ Se vea en: José Luis Acín FANLo (coord.), Armorial de Aragón (facs.), Zaragoza, 1997, fol. 314 .
} 
en algún momento pasó a formar parte del archivo familiar, que se integró en el de de los condes de Argillo, fondo en el cual se ha conservado hasta la actualidad. Este talegon debía contener todos los documentos referidos a la familia despositados hoy en el Archivo Histórico Provincial de Zaragoza, referidos sobre todo a la política matrimonial, los intercambios de propiedades y los testamentos, y fue la información básica utilizada en las historias del linaje realizadas en época moderna; aquí se debió integrar el cuadernillo que contiene este inventario, una vez que cumplió los fines prácticos para los cuales había sido redactado.

\section{EL NIVEL ECONÓMICO Y DE CONSUMO}

Más allá de los objetos asociados de manera más o menos evidente a la pertenencia a una determinada categoría social, en este caso a la nobleza local, con lo que ello acarreaba en una sociedad estratificada en función de la situación jurídica y no sólo económica, la mayor parte del ajuar nos está demostrando que los habitantes de esta casa disfrutaban de un cierto nivel de fortuna que marcaba sus capacidades de consumo. De hecho, aunque la sociedad aragonesa de finales del siglo XV estaba muy lejos del capitalismo, en ella la circulación y acumulación del dinero se había convertido en un factor fundamental de distinción social; la posesión de un determinado capital se manifiesta, entre otros elementos, en el disfrute de unas condiciones de existencia acomodadas, que se observan con bastante claridad en un inventario post mortem como el que nos ocupa.

En el texto aparece toda una serie de piezas que, por el material en el que estaban realizadas, tenían un valor muy elevado, básicamente en oro, plata y marfil. El uso de este tipo de materias tenía una doble función: por un lado se trataban de objetos de prestigio que tenían un claro cometido de ostentación, ya sea en la casa o en la indumentaria; por el otro, frente a bienes que se depreciaban conforme iban envejeciendo, éstos tenían un valor material intrínseco, por lo que su acumulación es, en realidad, una práctica de atesoramiento ${ }^{35}$. Este grupo de objetos valiosos se puede dividir en dos apartados: los utilizados como complementos decorativos de la indumentaria, y los que tenían algún tipo de finalidad doméstica. Entre los adornos personales aparecen: unos collares vermexos, una cadenica de oro, varias perlas, cuatro anyllos de plata - uno de los cuales tenía una piedra que parecía ser de unicornio, elemento al que se atribuían poderes mágicos-, y sobre todo un sillo de oro con las armas de Capata, pieza que por su valor material y simbólico debía ser lucido por el cabeza de familia, Sancho hasta 1484, y lo utilizaría para firmar sus cartas. Los objetos domésticos hechos en metal precioso son un salero, una copa y una docena de cucharetas, todo ello realizado d'argent. Es probable que esta vajilla no estuviera destinada al uso

\footnotetext{
${ }^{35}$ Christopher Dyer, Niveles de vida en la Baja Edad Media, Barcelona, 1991, p. 128.
} 
corriente en las comidas, sino a las ocasiones especiales y con invitados. También aparece un peyne de boyre (marfil) con letras de oro y varios fragmentos de oro dentro de un papel, que pesaban alrededor de un florín (en torno a tres gramos).

$\mathrm{La}$ indumentaria, como es bien sabido, resulta fundamental para la construcción de la imagen exterior del individuo, y es un indicador bastante fiable del rango social y económico; el vestido es un signo dentro de un código preestablecido ${ }^{36}$. Entre los atuendos de la familia Zapata aparecen algunos de uso corriente y valor no muy elevado, junto a otros que denotan la posición que disfrutaban. Los productos textiles eran núcleo de la industria artesanal bajomedieval y del comercio; al igual que en nuestros días, era un bien sujeto a los caprichos de las diferentes modas, y eran especialmente apreciadas ciertas prendas que venían de lejos a través de las rutas comerciales: en el inventario aparecen tejidos de Champaña, Holanda, Coutrai, Alemania, Arrás y Vizcaya, aunque en algún caso podrían ser imitaciones autóctonas que conservaron el nombre del producto original. En general se trata de productos de bastante calidad: entre las telas figuran algunas de lino y algodón, pero es la seda la que predomina, de precio mucho mayor que las anteriores; son prendas de colores diversos, y en algún caso se decoran con franjas de seda, pasamanerías o incluso botones de oro; junto con la procedencia, estos acabados constituyen otro indicativo de su calidad. Entre el vestuario femenino aparece ropa interior, como dos camisas y unas faldetas, dos trajes de lujo y un mongil negro, destinado posiblemente al luto; relacionado con la indumentaria de las mujeres, hay un mantico de criar ninyos, y varios complementos: un manto, una cofia y un vental o abanico, objeto valioso en aquel momento. El vestuario masculino consta de bastantes prendas de un cierto valor: dos peynadores, un sobrepeliz, un capuz, un jubón y varios elementos de la toca; en sentido contrario, figura un tavardo, tejido basto muy popular incluso en las clases humildes. Por último citaré algunas prendas que resulta difícil asignar a un género: dos zapatos, unas patoflas, un bonete o un almayçar, atavío habitual entre los mudéjares que se puso de moda en este periodo.

Los enseres domésticos y el nivel de confort que demuestran nos están hablando de la disposición de un aceptable nivel de consumo. El mobiliario destinado a dormir es un claro ejemplo: sólo figura una marfega, tela rellena de cáñamo o paja que hacía las veces de colchón, mientras que aparecen numerosas camas y camenyas equipadas con almadrach, lichera, lincuelos, delantelecho, cabecal y otros aditamientos relacionados con los lechos, que parecen adecuadamente provistos; los cofres que almacenan los objetos menudos estaban a menudo ferrados, muestra de solidez, y cubiertos por cuero o pinturas; había varios bancos, mesas y sillas, además de un escabelote para reposar los pies, en diferentes lugares de la casa y a veces con funciones

\footnotetext{
${ }^{36}$ Esta definición y numerosos datos sobre la indumentaria medieval han sido sacados de Cristina SIGÜENZA PELARDA, La moda en el vestir en la pintura gótica aragonesa, Zaragoza,
2000 .
} 
específicas, como las mesas de reparar y de comer. El conjunto de la casa estaba lleno de paramentos, bancales, coxines, cortinas, catifas, alfombras, etc. de formas y funciones muy diversas, que daban colorido y decoración a las diferentes estancias, y que en algunos casos estaban particularmente elaborados con decoraciones de pincel, que manifiestan la participación de artesanos o artistas locales en su ejecución. Por último hay que destacar que en una fecha relativamente temprana se encuentran unas oras d'enprenta scriptas en paper, cuya presencia indica que algún miembro de la familia estaba alfabetizado, aunque en comparación con las riquísimas bibliotecas privadas de otros inventarios aragoneses coetáneos resulta de gran pobreza: no parece que los Zapata tuviesen especiales inquietudes culturales ${ }^{37}$.

\section{CONCLUSIÓN}

La existencia de un inventario de los bienes de una residencia nobiliaria del Calatayud medieval nos permite conocer su cultura material con una precisión comparable a la que proporcionan las fuentes arqueológicas, y con la ventaja de reflejar el conjunto de los objetos, y no sólo aquellos que fueron abandonados y el paso del tiempo no hizo desaparecer. Por el contrario, a diferencia de la arqueología, la realidad fisica expresada por el inventario ha pasado por el filtro de su plasmación por escrito y en un código lingüístico en continua transformación: la relación entre significante y significado es, cuanto menos, problemática. Pese la debida prudencia que esta circunstancia impone, la información que suministra nuestro documento sobre los aspectos materiales es susceptible de ser usada en una interpretación más profunda de la familia Zapata, y de las actitudes y estrategias del grupo social al que pertenecía.

Pierre Bourdieu propuso en un conocido artículo la existencia de diferentes maneras de acumulación de capital: el capital económico, el capital cultural y el capital social ${ }^{38}$. Nuestro texto sólo refleja directamente la acumulación de capital económico en forma de un amplio conjunto de objetos materiales, algunos de los cuales tienen un considerable valor - se puede llegar a hablar de atesoramiento-. Sin embargo, un análisis un poco más atento de los bienes familiares, junto a los datos históricos esbozados al comienzo del artículo, muestran que los Zapata acumulaban capital de maneras bastante diversas. La familia mantenía relaciones horizontales con otros linajes de escuderos de la ciudad (Muñoz de Pamplona, Sayas, Forcén), y verticales, tanto con la importante familia de los Luna, como con una

\footnotetext{
${ }^{37}$ WITTLIN, Un inventario turolense de 1484, $\mathrm{pp}$ 187-188; Juan J. Fernando UTRILLA UTRILLA, Una biblioteca nobiliar aragonesa de mediados del siglo XV: inventario de libros de Alfonso de Liñán (1468), señor de Cetina (Zaragoza), en "Aragón en la Edad Media", 7 (1987): pp. 177-198; José Luis CORRAL LAFUENTE, La torre y el caballero. El ocaso de los feudales, Barcelona, 2002.

${ }^{38}$ Pierre BOURDIEU, Las formas del capital. Capital económico, capital cultural y capital social, en "Poder, Derecho y Clases Sociales", (2000), pp. 131-164.
} 
clientela a la que arrendaba tierras y que en caso de necesidad les acompañaría en el pequeño contingente armado que estaban en condiciones de equipar; todo este capital social debía ser cuidado, a lo que ayudaba el efecto "publicitario" del palacio con su ajuar, o la riqueza de la indumentaria. El capital cultural no tomaba la forma de una educación refinada y humanista $-\mathrm{o}$ al menos eso se deduce de la mediocridad de la biblioteca-, sino de la persisencia premeditada y arcaizante de una formación y valores caballerescos que, al fin y al cabo, eran los que daban sentido a la existencia e importancia del grupo social de la baja nobleza aragonesa. El capital económico de la casa de los Zapata tal vez no era lo bastante grande - al menos en el caso de esta ramapara justificar su predominio social, pero estaba orientado a reforzar las otras formas de acumulación de capital: aquellas que diferenciaban al escudero de quien no lo era, ¿acaso había mejor inversión que el refuerzo de su situación jurídica privilegiada?

El ajuar de la vivienda era en parte el resultado, pues, de una cierta estrategia en el juego social destinada a su propia reproducción dentro de las elites, pero hubo otros factores importantes: las necesidades básicas de los habitantes, la disponibilidad económica o unas circunstancias puntuales. Sin duda el gusto, facultad abstracta y resbaladiza, fue un elemento determinante a la hora de elegir un elemento u otro en los vestidos, la decoración o los muebles, pero eso no hace de ninguna manera arbitraria o caprichosa la cultura material, y es que, recurriendo de nuevo a Bourdieu, el gusto es una de las expresiones más refinadas y poco evidentes de la diferenciación social ${ }^{39}$.

En definitiva, en la casa de los Zapata de Calatayud todo estaba donde debía estar: es el reflejo consciente o no del orden social en la que se integraba.

\footnotetext{
${ }^{39}$ Pierre BouRdieU, La distinción. Criterio y bases sociales del gusto, Madrid, 1988.
} 


\section{APÉNDICE DOCUMENTAL}

1484, abril, 24 y 26. Calatayud.

Inventario de los bienes muebles de la vivienda del escudero Sancho Zapata, en Calatayud, realizado tras su fallecimiento por orden de los tutores de sus hijos $^{40}$.

AHPZ, Condes de Argillo, legajo 49, número 49. Cuadernillo de papel.

(2r) In Dei nomine, Amen. Noverint universi, et cetera, anno a nativitate Domini millesimo quadringentesimo octogesimo quarto, die videlicet connumerata vicesima quarta mense aprilis, apud civitatem Calataiubii, los magnificos Ysabel Capata, vidua, muger que fue de Joan Lopez de Villanova quondam, habitant en la dita ciudad de Calatayut, et Joan Capata, fijo de Sancho Capata, quondam, hermanos, scudero habitant en la ciudat de Caragoca, tutores et curadores testamentarios que se dixeron seyer ensemble con el magnifico Goncalvo Munyoz, mayor de dias, scudero habitant en el lugar de Morés, de las personas y bienes de Sancho, Goncalvo, Joan Capata, Ysabel Capata et Catalina Capata, hermanos pupillos, fijos de Sancho Capata, quondam, hermano de los dichos Ysabel Capata vidua et Joan Capata, tutores predichos, los quales dichos tutores atendido que ellos son tenidos fazer inventario segunt fuero, y el dicho Goncalvo Munyoz esta enfermo y no puede venir a fazer el dicho inventario con ellos ensemble, por tanto en absencia del dicho Goncalvo Munyoz, contutor con ellos, dixeron que (2v) por satisfacer al Fuero, presentes mi notario y testimonyos infrascriptos, procidian y procedieron a fazer $\mathrm{y}$ fazieron inventario de los bienes mobles del dicho Sancho Capata, quondam, que stavan en las casas de su habitación et se trobaban et trobaron dentro de aquellas al tiempo de su muerte. Las quales dichas casas son sitias en la dita ciudad de Calatayut, que afruentan con casas de Joan de Puydenina, con casas de Pero Tris, notario, et con casas de herederos de don Gostin de Savinyan quondam, en et dius la forma manera siguient e infrascripta:

Primeramente dentro de un palacio que sta en las dichas casas, de cara de la puerta, se trobaron los bienes siguientes:

Primo, una cama de fusta entaulada, con un colchon et dos lincuelos de cannyamo, et un delantelecho de canyamo con listas cardenas, et un cabezal blanquo de listas cardenas, et dos licheras, la una blanqua cardada et la otra de colores listada, et un banquo delante del lecho.

Item un paramento de cinco cortinas pintado de pinzel.

Item una cama de reposte chica donde duermen mocas, con un colchon de listas cardenas.

Item dos lincuelos de stopa.

\footnotetext{
${ }^{40}$ Para facilitar la comprensión del texto, he separado marcado con un espacio en blanco los cambios de habitación, y con medio espacio el comienzo de conjuntos diferenciados dentro de una misma estancia. Ninguna de estas separaciones está indicada en el texto original, por lo que deben ser tomadas con prudencia.
} 
Item una lichera de listas bermejas y amarillas e un racelexo de colores, traydo.

Item un cabeçal blanquo.

Item un cofre ferrado cubierto ( $3 r$ ) de cueros de buey, en el qual havia nueve lincuelos de canyamo de cada tres piernas.

Item dos ternas de lincuelos rasgados de canyamo.

Item un arcaz de noguera grande, en el qual havia unas tovajas con listas cardenas de coton.

Item tres tobaxas, las dos de canyamo e unas de stopa scacadas.

Item unas maseras de stopa con cordones.

Item unas tovajas rotas.

Item otro cofre ferrado cubierto de cuero en el qual havia los bienes siguientes:

Primo una copa d'argent sobredorada.

Item un salero d'argent con armas de capatas y lobos.

Item un cofretico chico de fusta.

Item un roquete de Chanpayna.

Item unas tovajas alamaniscas.

Item un pedaco de filo de seda y de oro, viejo.

Item un lincuelo obrado a los cabos de seda negra y bermeja de lino.

Item un peynador de Chanpayna obrado de seda de diversas colores randado y con botones de oro.

Item otro peynador de Olanda, obrado de seda blanca.

Item una toca de hombre de lino, en las orillas de vetas de seda.

Item un sobrepeliz rasgado de lienço vizcayno.

Item tres piecas de stopa de canyamo curadas, tirantes trenta y tres cobdos.

Item unas tovajas scacadas de lino.

Item una peceta de trapetes de boca, que eran seis.

Item dos trapetes, el uno de stopa y el otro de lino.

Item media pierna de stopa de canyamo.

Item (3v) unas oras d'enprenta scriptas en paper, cubiertas de cuero bermejo.

Item una cevadera de lana.

Item un talegon de cuero pleno de cartas y scripturas.

Item una spada guarnecida de negro.

Item otro cofre ferrado cubierto de cuero, en el qual havia dos tancaportas de amarillo e cardeno, con armas de los Capatas.

Item una lichera blanca cardada.

Item una sobremesa de verdura, con armas de los Capatas.

Item unos bancales de diversas colores listados.

Item un cofre pintado grande en el qual havia los bienes siguentes:

Primo un papallon de lienco vizcayno con listas negras.

Item un delantelecho de lienco vizcayno con listas negras.

Item otro delantelecho rexado con randas de desfila.

Item una banova de Champayna nueva.

Item quatro coxines enfundados, los dos de lienco vizcayno randados con randas de desfila.

Item los otros dos de Champayna encordados con cordon de grana.

Item dos camisas de muger de lienco vizcayno, todas listadas con listas, la una de liston leonado y la otra de liston verde.

Item tres linçuelos de Chanpayna de cada tres piernas.

Item unos manteles scacados de lino.

Item quatro pedaços de lienço de lino tirantes quinze coudos. 
Item un cofre pintado grande en el qual havia los bienes siguientes:

Primo unas faldetas de panyo pardillas layas con alorzas de muger.

Iem un brial encarnado con cortapisa de seda prieta forrado (4r) de panyo cardeno nuevo.

Item otras faldetas cardenas traydas de muger.

Item un manto de muger negro de Coutray.

Item un brial de muger blanquo de coton.

Item un mongil de muger negro, guarnecido de seda prieta.

Item un tavardo de hombre con mangas de panyo valenciano, guarnecido de passaman.

Item un pedaco de panyo encarnado.

Item un jubon de hombre de ceti con medias mangas de seda, de terciopelo negro.

Item dos trocetes de panyo negro.

Item un capuz frisonado de panyo negro.

Item un mantico de criar ninyos de panyo forrado de fusa amarilla.

Item otro capuz de burete traydo, de hombre.

Item un cofre; otro pintado con menuzeletes de los ninyos.

Item sobre un cofre crebado.

Primo un almadraque traydo con listas bemejas y blancas.

Item otro almadraque, blasco [sic] la cara y la forradura bermexa.

Item un cabecal con listas cardenas.

Item una lichera cardada blanca.

Item cinco piecas de bancales de armas reales y otras colores traydos.

Item otra pieca de bancales de lana de diversas colores.

Item cinco cortinas de un paramento pintado de arvoleda de pincel.

Item dos cortinas viejas de pincel.

Item dos catifas de piedes viejas.

Item otras dos piecas de bancales de colores.

Item una lichera blanqua de listas, vieja.

Item otra lichera blanca cardada.

Item una cortinica vieja rasgada.

Item una alhombraca de diversos colores.

Item un trapo $(4 \mathrm{v})$ de paret vermexo grande con armas de los Capatas.

Item cinco coxines de Raz y de catifas de asentar viejos.

Item dos mesas de fusta con sus piedes.

Item cinco cadiras de fusta.

Item un escabelete.

Item una caxa grande de noguera en la qual havia una lichera blanca cardada.

Item una catifa de colores.

Item cinco fundas de coxines pora dormir.

Item dos banovas de Champayna planas traydas.

Item quatro varas de cara de almadraque de coton cardeno y blanco.

Item dos cabecales obrados de seda de diversas colores del tiempo viejo.

Item unos tovaxones de stopa de lino, menos de dos cobdos.

Item una tovallola laurada de seda.

Item una pieca de bancales de brocado.

Item siete varas de manteles de stopa de canyamo.

Item defuera de la caxa dos piecas otras de bancales de brocado.

Item una terna de lincuelo de lino viejo.

Item un coxinico obrado de seda chico.

Item una spada vieja colgada en la paret. 
Item un candelero colgado de fusta en medio del palacio.

Item una cortina puesta en la paret del palacio de cardeno con ramos blancos vieja.

Item en el porche una alfacera puesta en la paret nueva.

Item otra alfacera vieja.

Item un drecador.

Item seis pavesses grandes pintados viejos.

Item un gamellon grande.

Item un arquivan (5r) grande viejo.

Item en una cambra una mesa grande de noguera con piedes pora tener costales de farina.

Item una artesa de masar chica.

Item dos capatos.

Item una cesta grande de canyas y bimbres.

Item unos almarios grandes pintados.

Item quatro paveses y un dargon pintados de fusta.

Item dos tablas de fusta de reparar.

Item una sarten mala et una cobertera de fierro.

Item un plato de stanyo grande.

Item unas asaderas de fierro.

Item dos calderos, el uno grande y el otro chico cabientes tres cantaros poco mas o menos.

Item dos trehudes de fierro las unas crebadas y las otras buenas.

Item en una cambreta una camenya de fusta de carruchas el suelo de canyas en la qual ay un almadrach de listas cardenas traydo.

Item dos lincuelos de stopa de canyamo.

Item una lichera con listas et un racel de colores traydos.

Item un cabecal del tiempo viejo obrado de seda vermexa traydo.

Item en una cambreta dos piedes con tablas con una marfega.

Item quatro almadraques, los tres de listas cardenas y el uno blanquo.

Item una lichera blanca con listas vermejas trayda.

Item un caxon amarillo de fusta.

Item una caxa de pino en la qual havia una tovallola (5v) de lino de desfilado.

Item unas tovachicas de canyamo chicas de un coudo.

Item una cofia et un peynador de lienco vizcayno.

Item una ropa de panyo negro forrada de penya negra.

Item un bonete negro doble de panyo.

Item un delante cama de stopa de lino.

Item una sobremesa de armas reales.

Item una tovallola de lino.

Item otra tovallola de lino.

Item un pedaco de seda negra vieja.

Item un cabecal obrado de seda vermexa del tiempo viejo.

Item un almayçar traydo.

Item una limpiadera.

Item unas unas patoflas viejas.

Item un cofre ferrado en el qual havia los bienes siguientes:

Primo una causa pequenya pintada en la qual havia dos beatillas de seda.

Item un cuerpo de seda mixta con listas cardenas, guarnecido de argenteria.

Item otra beatilla de coton.

Item un trapo de cuello de seda.

Item un coudo de lienco vizcayno. 
Item dos trapos de cuello de filo de oro.

Item dos cuentas, las unas de bellotas y las otras de açavacho.

Item un stutx.

Item granadura de dos o tres colores.

Item unos collares vermexos con una bolsica d'oro.

Item unas frangas de seda vermexas.

Item unos pedacos de argenteria de trapos de cuello.

Item en otra causa, quatro anyllos de plata con ciertas piedras, en la qual ay una que parece olicorni.

Item en un paperico una verguica de oro y unos pedacicos d'oro, pessante todo fasta un florin.

Item un sillo de oro con las armas de Capata, fasta pesso (6r) de tres florines.

Item dotze granados de plata de unos botones del tiempo viejo.

Item tres tovallolas de lienço vizcayno con sus franjas de seda.

Item una caxita de arambre con cinco perlas y una cadenica de oro.

Item un cuerpo de muger de Olanda.

Item un trapo de boca.

Item una dotzena de cucharetas d'argent.

Item un peyne de boyre nuevo con letras de oro.

Item otra causida con un trapo de ciello de seda.

Item un vulto de filo de argenteria fasta unas tres onzas.

Item un vental negro de dona.

Et de las sobre dichas cosas, los sobredichos tutores dixeron que, por quanto no havian podido acabar el dicho inventario el present dia, que requirian et requirieron del sobredito inventario seyer fecho [ante] publico testimonios. Fueron a las sobreditas cosas presentes Domingo Munyoz y Lois de Forcen, vecinos y habitantes de la dita ciudad de Calatayud.

Apres de aquesto dia, es asaber, que se contava y conta vinte y seis dias del sobredicho mes de abril, en la dicha ciudat de Calatayud, et dentro de las sobredichas casas de part de suso confrontadas, los sobredichos Isabel Capata et Joan Capata, tutores predichos, continuando el dicho su inventario, presentes mi dicho notario et testimonyos $(6 \mathrm{v})$ infrascriptos, procedieron a inventariar et fizieron inventario de los bienes restantes, los quales inventariaron en la forma siguiente:

Et primo, en una retreta de la sala de las dichas casas se trobaron los bienes siguientes:

Item una manta grande de paret de diversas colores con armas de los Capatas.

Item dos piecas de bancales de diversas colores.

Item una tabla con sus piedes.

Item una cama de fusta entaulada.

Item quatro candeleros de allaton.

Item un banquo.

Item un retablico de fusta pintado.

Item en otra retreta de la sala, una cama de fusta entaulada.

Item en la sala un almadraque blanquo con listas cardenas.

Item una lichera blanca con listas bermejas.

Item un banco con sus piedes.

Item quatro manteles, los dos de stopa de canyamo y los otros de companya de canyamo.

Item diez panyecillos de boca. 
Item dos tovallolas de lino de manos.

Item otras dos tovallolas de stopa de lino.

Item tres savanas de lino.

Item quatro savanas de canyamo.

Item tres savanas de stopa.

Item un parador grande de fusta.

Item en una cambra los bienes siguientes:

Primo quatro ollas de cobre.

Item tres calderos grandes y un olla d'arambre.

Item tres bacines.

Item unas trehudes grandes $(7 \mathrm{r})$ y otras chicas colgaderas.

Item tres fogariles de fierro pora tener brassa.

Item dos fogariles pora cozinar de tierra, el uno crebado.

Item un calderico de fierro.

Item unas llares con tres baras de fierro grandes pora parar.

Item dos asaderas pora ad asar al fuego.

Item unas pressiones de fierro largas.

Item un porpalo de fierro.

Item dos candeleros grandes de fierro pora sala de tener munchas candelas.

Item un restillo grande de lino con dos copas.

Item dos fajas pora ombres d'armas.

Item un pesso grande de fierro et un otro chico con una romania.

Item un candelero grande de fierro.

Item un piet de devanaderas de fierro.

Item tres tornos de ballestas de fierro.

Item tres ballestas de fusta.

Item una ballesta de azero.

Item dos pares de cubiertas de cavallo, las unas con clavellinas y las otras de verde y leonado.

Item cinquo assadores de fierro y dos grayllas, la una como asador.

Item una sarten y una padilla d'arambre.

Item una cuchara de fierro y un candil.

Item unos ganchos de fierro e unos peynes.

Item palafrenes y una calderica chica de arambre.

Item un asnico de fierro pora el fuego, a tener la lenya.

Item una caxa crebada abier(7v)ta con riendas y falsas riendas viejas.

Item una silla vieja.

Item otros dos palafrenes.

Item una caxa vieja con armas viejas.

Item otro restillo chico viejo.

Item dos medias de mesurar trigo.

Item un cuenco de medir vino.

Item tres terrizas de tierra grandes crebadas.

Item ocho tinajas crebadas.

Item una legona.

Item una pala de forno de fierro.

Item una tabla grande de comer sinse tornillo.

Item otra tabla grande de comer.

Item una cuna et una cadira de barvero grande.

Item un cofre pintado con unos quixotes y canyones y un casquete.

Item dos artesas viejas crebadas. 
Item unas arguinas.

Item un caxon de botica.

Item una cesta grande en dos roscadas.

Item una caxuela crebada.

Item unas curacas blanquas de chamuç.

Item un cofre en el qual havia dos pares de curaças guarnecidas de negro, et un capacete, y una banera, y unas guardas con sus canyones, et unas vias de malla de piernas.

Item otras curaças guarnecidas de negro.

Item unos gozetes.

Item una cervellera y un guante de malla.

Item un fierro de lança.

Item una aljava con nueve passadores.

Item dos (8r) piedes de tornos crebados.

Item quatro orcas chicas y grandes e dos charros blancos.

Item un almadrach blanco viejo.

Item una cortina pintada de pincel con personages.

Item dos rateles viejos y unos bancales viejos.

Item un coxin de possar de raz.

Item otro racel viejo y roto e dos cabecales, el uno blanco y el otro de listas cardenas.

Item una pieca de tovachones de stopa de lino por curar tirante seis cobdos y medio.

Item una pieça por curar de setze trapetes de boca de stopa de lino.

Item una cesta en donde havia cuarenta y quatro ovillos de canyamo.

Item un enbudo de fusta.

Item una caldera chica con cal.

Item dos banovones rasgados viejos, el uno de listas cardenas y el otro blanquo.

Item en la cozina una sarten con su rasera y tres asadores de fierro.

Item dos cucharas y la una de provar.

Item un rallo de fierro.

Item un mortero de allaton con su mano.

Item una tabla con sus piedes puestos en ella de comer.

Item una tinaja de agua.

Item una padilla de arambre.

Item dos bancos et un parador viejo.

Item en el reposte un cofre ferrado de tener pan.

Item dos toneles de tener vino.

Item quatro tinajas.

(8v) Item en la bodega un tino grande et onze cubas, las nueve grandes de las quales havia quatro grandes plenas de vino tinto.

Item tres toneles plenos de vino blanquo y el un tonel es de Goncalvo Munyoz, excepto el vino.

Item un pozal de sacar el vino y un par de artesas, la una chica y la otra grande.

Item un leguado de cubas de sparado.

Item onze cuevanos de vendemar.

Item una tinaja de agua.

Item dos enbudos de la bodega, el uno chico y el otro grande.

Item en el porje quatro talegas y un costal.

Et de todas y cada una cosas sobreditas, los dichos Ysabel Capata e Joan Capata, tutores y curadores predichos, requirieron a mi, dicho e infrascripto notario, 
les ende fiziese y testificase carta publica a scargo d'ellos, et a conservación de los dichos pupillos actum ut supra. Testimonyos fueron a las sobreditas cosas presentes los sobreditos Domingo Munyoz y Lois Forcen, vezinos siquiere habitantes de la dicha ciudat de Calatayut.

Sig [signo] de mi, Joan Cortes, habitant en la ciudad de Calatayut, por (9r) actoridat real notario publico por los regnos de Aragon y de Valencia, qui a las sobredichas cosas ensemble con los sobredichos testimonyos present fue y aquellas scrivi et cerre. Constame de raso corregido en do es scripto "mo".

Fecha de recepción del artículo: septiembre 2007.

Fecha de aceptación y versión final: mayo 2009. 